International Journal of Poultry Science 8 (6): 559-564, 2009

ISSN 1682-8356

(C) Asian Network for Scientific Information, 2009

\title{
Simulation Models for Estimating Optimal Vaccination Timing for Infectious Bursal Disease in Broiler Chickens in Paraguay
}

\author{
K. Suzuki ${ }^{1}$, J. Caballero' ${ }^{2}$ F. Álvarez ${ }^{2}$, M. Faccioli ${ }^{2}$, M. Goreti ${ }^{2}$, M. Herrero' and M. Petruccelli' \\ 'Laboratorio de Diagnóstico de Enfermedades de las Aves y los Pilíferos, \\ Facultad de Ciencias Veterinarias, Universidad Nacional de La Plata, Argentina \\ ${ }^{2}$ Laboratorio de Diagnóstico de las Enfermedades de las Aves, Facultad de Ciencias Veterinarias, \\ Universidad Nacional de Asunción, Paraguay
}

\begin{abstract}
This study shows the results of estimating optimal vaccination timing for infectious bursal disease in broiler chickens in Paraguay, using spreadsheet simulation models. Fourteen flocks of broiler chicks were kept under observation. Sera were collected from randomly-selected 20 chicks per flock at 1, 4 and 8 days of age, and assayed by a commercial Enzyme-linked Immunosorbent Assay (ELISA) to evaluate Maternally Derived Antibody (MDA) titres. Deterministic (an age-based estimation method called the Deventer formula) and stochastic (through inclusion of uncertainty in the parameters) models were developed with the data. In the deterministic models, all the estimated optimal vaccination timings of each flock at the three sampling time points were between 16 and 24 days of age. In the stochastic models, each of the median optimal vaccination timings was estimated later than the corresponding point-estimate timing, generated by the deterministic version. Uniformity of the MDA titre distribution in the flocks was considered in relation to the number of vaccinations required. The ELISA results provide only a rough indication, in the case of deterministic model in particular. A stochastic version of the same model, in conjunction with the use of a concept of uniformity might give a solution to the problem.
\end{abstract}

Key words: Gumboro disease, modelling, South America

\section{INTRODUCTION}

Infectious Bursal Disease (IBD) Virus (IBDV) is the etiological agent of "Gumboro disease" which is a serious cause of economic losses in the poultry industry (Lukert and Saif, 2003). Antibody detection is a practical tool in the prevention against IBD. It is utilized to estimate the optimal vaccination days of age when measuring the status of Maternally Derived Antibody (MDA) in chicks. Vaccination in the existence of MDA levels above the breakthrough titre of the vaccine is unsuccessful as this will induce neutralization of the vaccine (De Wit et al., 2001). On the other hand, the chicks should not be left unvaccinated longer than necessary. It is, therefore, of the utmost importance that the optimal vaccination timing can be ascertained.

The MDA titres measured by Virus Neutralization (VN) have a constant decline rate with age (Berg and Meulemans, 1991). This permits to estimate the optimal vaccination timing on the basis of serology in day-old chicks. However, this sensitive test is time-consuming and expensive to be used on a routine basis. Meanwhile, Enzyme-linked Immunosorbent Assay (ELISA) is relatively less expensive and results are acquired quickly and normally constant. Furthermore, titres of this assay may correlate well with VN titres (De Herdt et al., 2005). Thus, ELISA is usually used to estimate optimal vaccination timing in the field (De Wit et al., 2001). Several formulas for estimating the optimal days of age for vaccination for a specific flock, such as Kouwenhoven formula and Deventer formula, are used for routine application (De Wit, 2001; Kouwenhoven and Van den Bos, 1994).

Previous researchers have examined the age-based estimation method for IBD vaccination in chicks described above and tried to improve the method in conjunction with several different methods, such as the possible use of ELISA Sample-to-positive (S/P) values into MDA levels (De Herdt et al., 2005) and the rate of weight gain in chicks (Vaziry et al., 2007). Nevertheless, a stochastic model closely connected with the concept of the age-based estimation method through inclusion of uncertainty in the model parameters has not been published. In Paraguay, South America, vaccination programmes with recommended timing for IBD vaccination provided by foreign vaccine manufacturers are commonly used, which are not always adapted to the specific conditions on the poultry farm and to the status of MDA in chicks. The objective of this study was to estimate the optimal days of age for $I B D$ vaccination using deterministic (original Deventer formula) and stochastic models, with data derived from a field investigation of broiler farms in Paraguay.

\section{MATERIALS AND METHODS}

Field data collection: Paraguay is a landlocked country with a land area of $406,752 \mathrm{~km}^{2}$, located in the centre of 
South America, with the current estimated population of 6.2 million people. Paraguay has a poultry population of 17 million, a poultry meat production of 37,000 tonnes per year and a poultry egg production of 100,000 tonnes per year (FAO, 2001; FAO, 2008).

For logistic reasons, 14 flocks of broiler chicks (Cobb breed) derived from breeder flocks belonging to the same hatchery were kept under observation. They were monitored from March to July 2007. All the study poultry farms routinely used both a combination of hot and intermediate vaccine for IBDV control. Nothing has been reported on clinical symptoms of an IBDV infection for at least a year. Sera were collected from randomlyselected 20 chicks per flock at 1, 4 and 8 days of age, and assayed by a commercial ELISA (FlockChek IBD, IDEXX Laboratories Inc., Westbrook, ME, USA) as indicated by the manufacturer instruction. None of the chicks had been vaccinated against IBDV prior to sample collection. Data collected were entered into a database using the Base in the Openoffice. org software version 2.4.1 (Sun Microsystems, Santa Clara, CA, USA). Data were checked for normality and the MDA values were logarithmically transformed ( $\log _{2}$ scale) as "Ig (MDA)". Box-and-whisker plots of the log-transformed MDA values with the 14 flocks and three sampling time points were drawn for visual assessment. In order to evaluate uniformity of the MDA titre distribution of each study flock, Coefficient of Variation (CV) at the three sampling time points were calculated. These $C V$ values were compared with the CV benchmark of $30 \%$, assuming a flock with less than this percentage has a good uniformity of the MDA titre distribution. If the uniformity of the MDA is good (CV $<30 \%)$, only one vaccination is required. If the uniformity is poor $(\mathrm{CV}=$ $30 \%$ ), more than one vaccination are required (Intervet, 2003).

Deterministic model development: The Deventer formula was employed to estimate the optimal age for vaccination for a specific flock based on the MDA level, its variation (relates to $\mathrm{CV}$ described above), the genetic background of the chicks (relates to half-life described below), and the IBD vaccine strain (relates to breakthrough titre of the vaccine described below). Its calculator using spreadsheet software Microsoft Excel 2003 (Microsoft Corporation, Redmond, WA, USA) was freely available through the Internet (Intervet, 2003). The principle was as follows (De Wit, 2001).

Optimal age $=\quad$ half-life ${ }^{*}$ Ig (MDA titre chick\%) $-\lg$ (breakthrough)\} + age at sampling + correction

where:

half-life $=$ half-life value of the MDA in the type of chicks being sampled (e.g. 3 days for broilers)

MDA titre chick\% = MDA titre of the chick representing a certain percentage of the flock (e.g. $75 \%$ as a default percentage of the calculator)

breakthrough $=\quad$ breakthrough titre of the vaccine being used (e.g. 125 for an intermediate vaccine)

age at sampling $=$ age of the chicks at sampling correction $=\quad$ extra $(4-x)$ days when the sampling was conducted at $x$ days of age $(x=0,1,2,3)$

Using the Deventer calculator spreadsheet, optimal timings for IBD vaccination of each study flock at the three sampling time points were estimated. The values such as half-life of 3 days, MDA titre chick $\%$ of $75 \%$ and breakthrough of 125 were adopted for calculation. Flocks with less than 18 samples were excluded for further calculation due to less reliability of the estimates (De Wit, 2001).

Stochastic model development: The Deventer calculator spreadsheet in combination with the simulation add-in software @RISK 5.0 for Excel (Palisade Corporation, Newfield, NY, USA) were used to construct the stochastic models through inclusion of uncertainty in the model parameters. The built-in Distribution Fitting function in the software was firstly used to fit probability distributions to each MDA titre dataset of the study flocks at three sampling time points. In order to select the most appropriate probability distribution, Anderson-Darling goodness-of-fit test was used (Law and Kelton, 2000). The deterministic model described above was converted into a stochastic model by representing parameters "half-life" and "breakthrough" in the model, which was considered to be subject to variation, with the following probability distribution.

half-life $\sim$ Normal (mean $=3.25$, standard deviation $=$ 0.1275 )

breakthrough $\sim$ Normal (mean $=125$, standard deviation $=6.25$ )

Input parameter values for defining the probability distributions above were obtained from De Wit (2001). Monte Carlo simulation was used to randomly select values from probability distributions. Individual simulations consisted of 10,000 iterations which were considered more than sufficient to ensure convergence of the output distributions. Cumulative probability distributions for estimated optimal days of age for IBD vaccination of each study flock at the three sampling time points were illustrated. In order to investigate the relative importance of the $\mathrm{CV}$ related to MDA titres for deciding the number of vaccinations, flocks with the largest and smallest $C V$ at 1 day of age were selected respectively. The output from all iterations was used to 
Int. J. Poult. Sci., 8 (6): 559-564. 2009

generate probability distributions of estimated optimal days of age for each percentage of the flocks responding IBD vaccination. The differences of estimated optimal days of age between corresponding to $75 \%$ and to $25 \%$ of the two selected flocks were considered, respectively.

\section{RESULTS}

Field data: Table 1 presents the structure of the data. The missing data accounted for $3.0 \%$ for the dependent variable. On visual assessment using Fig. 1, the logtransformed MDA values of the study chicks were variable between individuals as well as flocks. Temporal downward trends of the MDA values from Day 1 to Day 8 for each flock were also observed. Fifty percent $(95 \% \mathrm{Cl}$ : $27-73 \%)$ of the flocks had a mild elevation in median MDA values from Day 1 to Day 4. The MDA data of Flock $B$ and $J$ at Day 4 and 8 were excluded for further calculation, as they did not fulfil a criterion (no. of chicks $=18$ ) described above. Forty-three percent ( $95 \% \mathrm{Cl}: 21$ $67), 42 \%$ (95\% Cl: $19-68)$ and $25 \%(95 \% \mathrm{Cl}: 9-53)$ of the

Table 1: Structure of the data from 815 samples from broilers in Paraguay in 2007

\begin{tabular}{|c|c|c|c|}
\hline \multirow[b]{2}{*}{ Flock } & \multicolumn{3}{|c|}{ Number of samples recorded } \\
\hline & Day 1 & Day 4 & Day 8 \\
\hline $\bar{A}$ & 19 & 19 & 19 \\
\hline B & 20 & 17 & 17 \\
\hline c & 20 & 20 & 20 \\
\hline D & 20 & 20 & 20 \\
\hline $\mathrm{E}$ & 20 & 20 & 20 \\
\hline $\mathrm{F}$ & 20 & 20 & 20 \\
\hline G & 20 & 20 & 20 \\
\hline $\mathrm{H}$ & 20 & 20 & 20 \\
\hline 1 & 20 & 20 & 18 \\
\hline J & 18 & 16 & 15 \\
\hline K & 19 & 19 & 19 \\
\hline L & 20 & 20 & 20 \\
\hline$M$ & 20 & 20 & 20 \\
\hline $\mathrm{N}$ & 20 & 20 & 20 \\
\hline Total & 276 & 271 & 268 \\
\hline
\end{tabular}

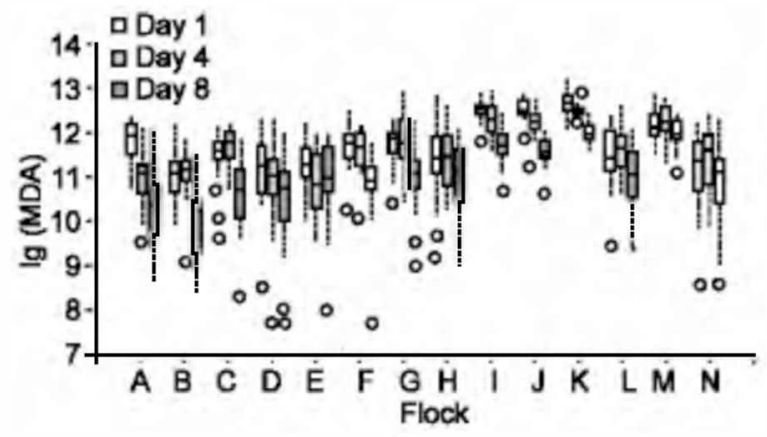

Fig. 1: Box and whisker plots of $\lg$ (MDA), with the 14 study flocks (A-N) and three sampling time points (Day 1, 4 and 8), based on data from 815 samples from broilers in Paraguay in 2007

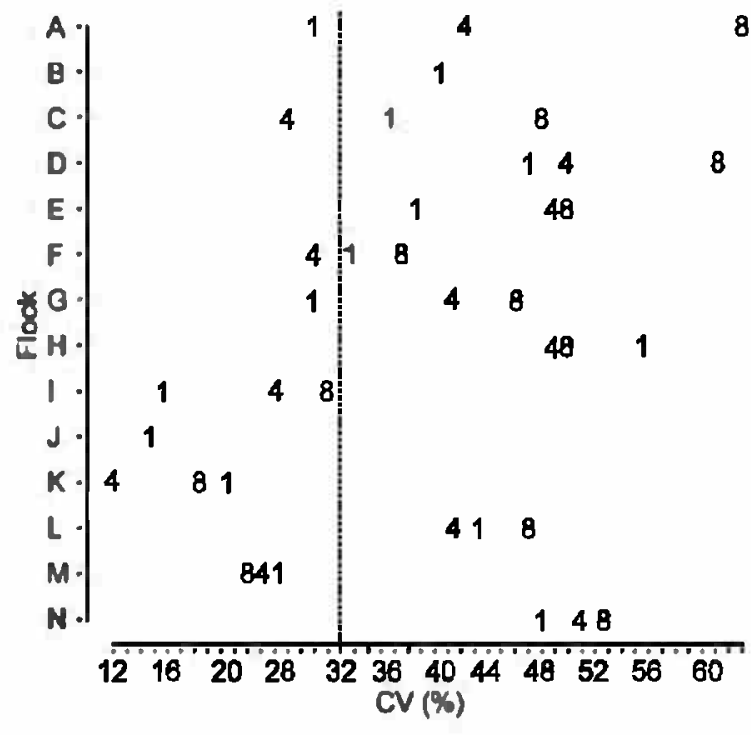

Fig. 2 Dotplot for different sampling time points (1 = Day 1, $4=$ Day 4, $8=$ Day 8 ) presenting the relationship between the $\mathrm{CV}$ and study flocks. Vertical dashed line corresponds to the CV value of $30 \%$

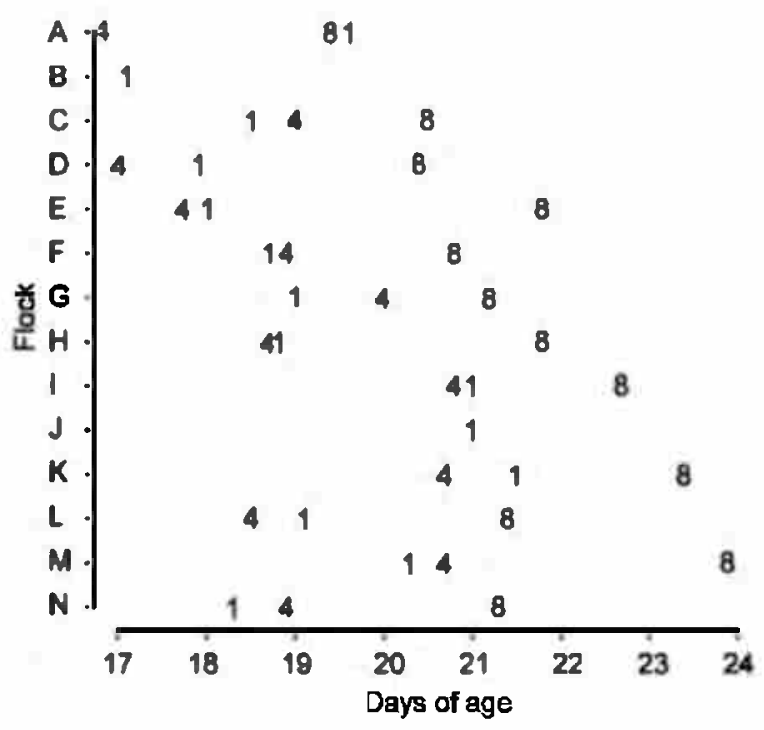

Fig. 3 Dotplot for different sampling time points (1 = Day 1, $4=$ Day 4, $8=$ Day 8 ) presenting the relationship between the estimated optimal days of age corresponding to $75 \%$ of the flock responding IBD vaccination and study flocks, assuming the use of an intermediate vaccine with a breakthrough titre of 125

flocks had a good uniformity of the MDA titre distribution (CV $<30 \%$ ) at Day 1, 4 and 8 , respectively (Fig. 2)

Deterministic model: All the estimated optimal vaccination timings of each study flock at the three 
Int. J. Poult. Sci., 8 (6): 559-564, 2009
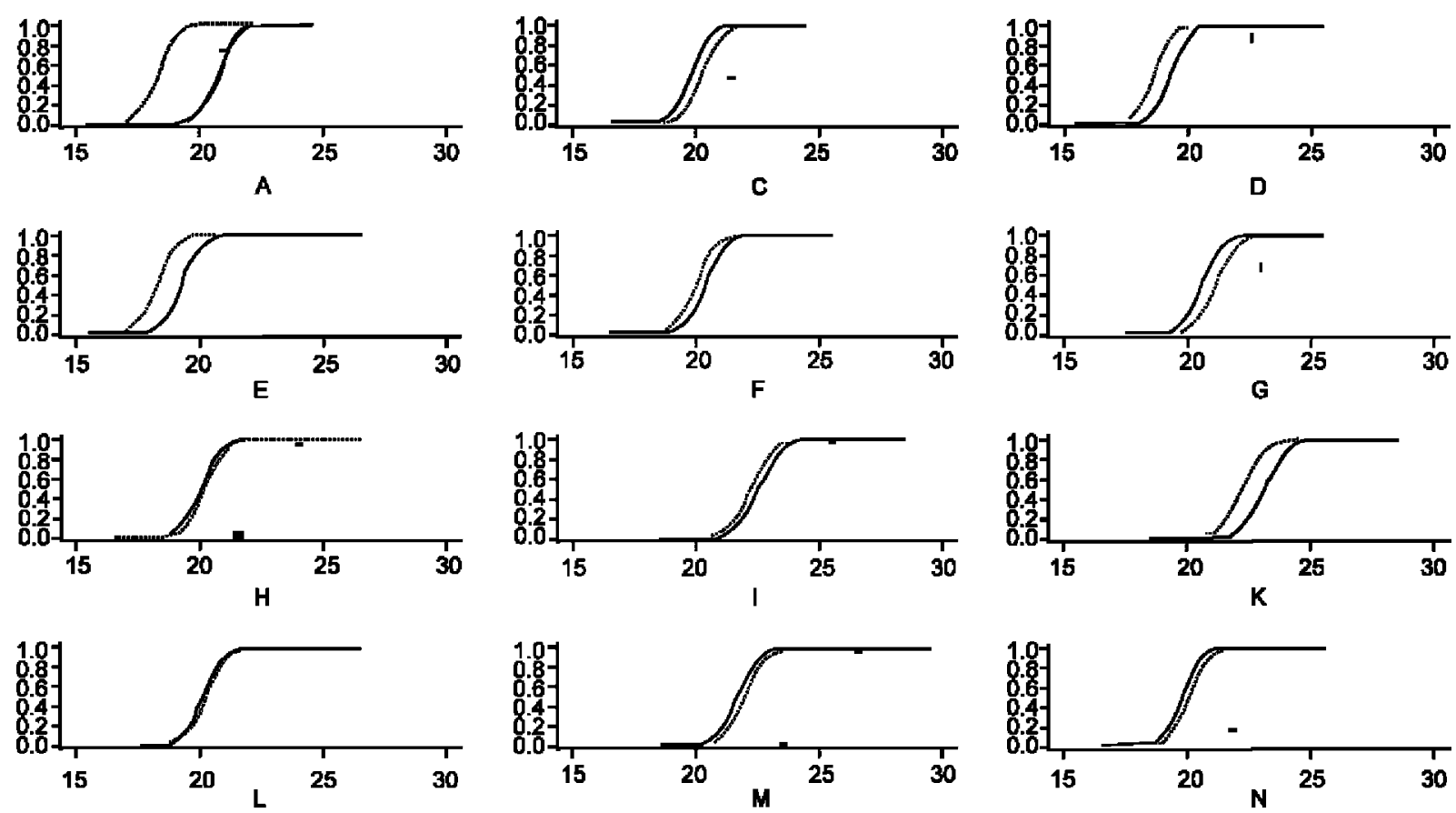

Fig. 4: Graphs with cumulative probability distribution value on the $y$-axis and estimated optimal days of age corresponding to $75 \%$ of the flock responding IBD vaccination on the $x$, for the 12 study flocks (A - N, except for $B$ and $\mathrm{J}$ ) and three sampling time points (solid line = Day 1, dashed line = Day 4, dotted line = Day 8), assuming the use of an intermediate vaccine with a breakthrough titre of 125

sampling time points were between 16 and 24 days of age (Fig. 3). Ninety-two percent ( $95 \% \mathrm{Cl}$ : $65-99)$ of the flocks at Day 8 had a later estimated timing than those at Day 1 and Day 4.

Stochastic model: Figure 4 represents the models with inclusion of uncertainty in the parameters. Each of the median optimal vaccination timings was later than the corresponding point-estimate timing, generated by the deterministic version of the same model. Figure 5 summarizes the probability distributions of the estimated vaccination timing for each percentage of the two selected flocks (Flock I and $H$ ). The Flock I had a good uniformity of the MDA with CV of $16 \%$, therefore only one vaccination would be required at 22 days of age. On the other hand, Flock $H$ was an example of poor uniformity with $\mathrm{CV}$ of $54 \%$. It would first be given to immunize the chicks with low MDA titres (25\% of the chicks) at 16 days of age and a second dose was required to protect the high-MDA chicks (75\% of the chicks) around four days later.

\section{DISCUSSION}

Field data: The ELISA kit used in this study is one of the recommended commercial kits for taking advantage of the Deventer formula. If the formula is used for other ELISAs without approval which requires comparison of the half-life to $V N$, reproducibility of measured results and etc., discrepancies will occur (De Wit, 2001). In considering the use of the Deventer formula, a minimum of 18 chicks per flock at each different sampling time point were required to obtain a representative sample of the flock (De Wit, 2001). In this study two flocks at two different sampling time points were excluded due to missing data items. Missing data are a major source of bias. However, missing data are inevitable in routinely collected data and reflect the quality of data collection. There is potential for its improvement to adopt computer intensive re-sampling method (Efron, 1979), based on the probability distributions fitted by the built-in Distribution Fitting function although this method is not used in this study.

Seven out of 14 flocks showed a slight increase in median MDA values from Day 1 to Day 4 . This can be partly explained by the fact that absorption of yolk compensates for the decrease of titre by metabolism and growth (De Wit, 2001). It seemed less likely to have a good uniformity ( $\mathrm{CV}<30 \%$ ) of the MDA titre distribution in the flocks across the three sampling time points (Fig. 2). However, it is not possible to draw definitive conclusions in relation to deciding the number of vaccinations, because of relatively broad $95 \% \mathrm{Cls}$ for proportions of the flocks with a good uniformity at each sampling time point. 
Int. J. Poult. Sci., 8 (6): 559-564. 2009
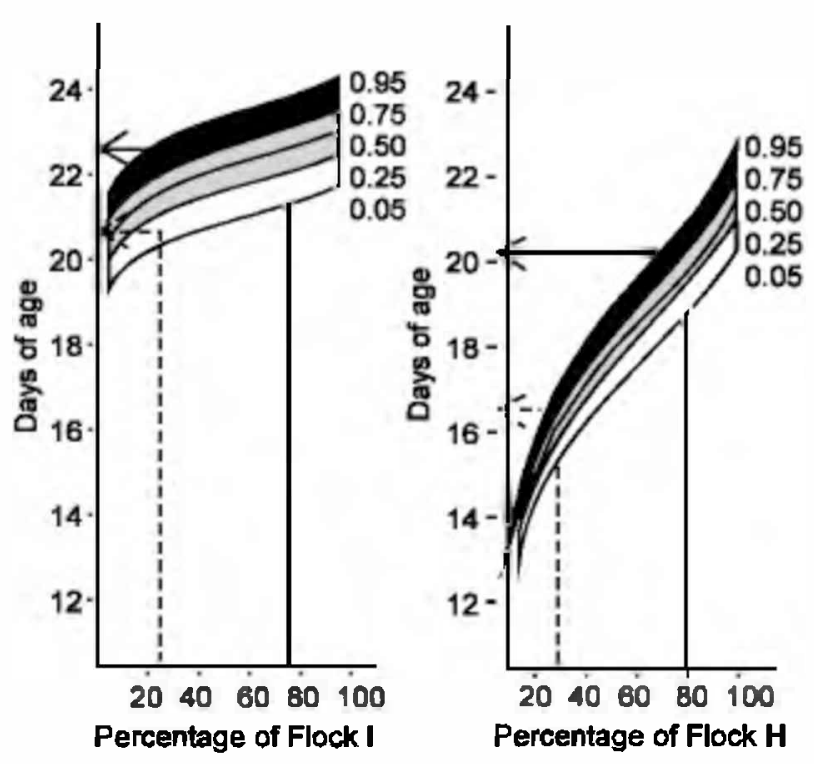

Fig. 5: Summary graphs presenting the median and $5^{\text {th }}, 25^{\text {th }}, 75^{\text {th }}$ and $95^{\text {th }}$ percentiles of the estimated optimal days of age for each percentage of the flock responding IBD vaccination using MDA data at Day 1 [Flock I with $\mathrm{CV}$ of $16 \%$ (left), and Flock $\mathrm{H}$ with $\mathrm{CV}$ of $54 \%$ (right)], assuming the use of an intermediate vaccine with a breakthrough titre of 125. Solid and dashed arrows indicate the median estimated optimal age corresponding to $75 \%$ and $25 \%$ of the flock responding IBD vaccination, respectively

Determ inistic model: The estimated optimal vaccination timings were in broad agreement with published range of the values from 14-21 days of age (Lukert and Saif, 2003). The estimated optimal ages for IBD vaccination of each flock at Day 1 nearly accorded with those at Day 4. In comparison with the estimated optimal ages at Day 8 , however, the MDA titres at Day 1 and 4 may produce earlier vaccination age estimations on the basis of ELISA. It was known that poorer results were acquired when vaccination age estimations were produced on the basis of 1-day-old chicks with high antibody titres (De Herdt et al., 2005). In spite of a good correlation between mean ELISA titres and VN values, there was little correlation between absolute values in the two tests (De Wit, 2001). Therefore, this might mostly be the result of the fact that absolute values of MDA titres in the higher titre range provided by ELISA are considerably lower than those measured in VN. On account of this, chicks with high antibody titres at Day 1 and 4 could be considered susceptible to vaccine at too early an age. Therefore, these data show that advice on the optimal age of IBD vaccination on the basis of ELISA results should only be considered as a rough indication.
Stochastic model: The results of the stochastic models demonstrated that optimal vaccination timings estimated by the deterministic models were likely to be at early an age. Although the use of MDA data at Day 1 for estimating optimal vaccination timing has a drawback described above, it is easier that collecting samples in the hatchery at Day 1 in comparison with that in the farm at later days of age, as the specification of the different breeder flocks can be traced with no trouble. Because of this, MDA data at Day 1 were selected to look into the importance of the $C V$ related to MDA titres. The Flock I had the best uniformity of the MDA in the study flocks, which should be vaccinated only once at 22 days of age, based on the current assumption for vaccination of this study. If the $C V$ value related to MDA titres is large such as Flock $H$, it may be recommended to vaccinate twice (with a couple of days interval) or to vaccinate early with a hot vaccine (in the presence of a high field antigenic pressure).

Conclusion: In conclusion, the ELISA still can be a practical tool in estimating IBD vaccination timing. The ELISA results, however, provide only a rough indication, in the case of deterministic model in particular. A stochastic version of the same model including uncertainty in the model parameters, in conjunction with the use of $\mathrm{CV}$ value might give a solution to the problem.

\section{ACKNOWLEDGEMENT}

This study was carried out as part of the project for the capacity development for improvement of livestock hygiene in the southern part of South America through regional cooperation [in Spanish: Proyecto de desarrollo profesional continuo para los veterinarios del Sur (PROVETSUR)], funded by the Japan International Cooperation Agency.

\section{REFERENCES}

Berg, T.P. and G. Meulemans, 1991. Acute infectious bursal disease in poultry: protection afforded by maternally derived antibodies and interference with live vaccination. Avian Pathol., 20: 409-21.

De Herdt, P., E. Jagt, G. Paul, S. Van Colen, R. Renard, C. Destrooper and G. Van den Bosch, 2005. Evaluation of the enzyme-linked immunosorbent assay for the detection of antibodies against Infectious Bursal Disease Virus (IBDV) and the estimation of the optimal age for IBDV vaccination in broilers. Avian Pathol., 34: 501-504.

De Wit, J.J., 2001. Gumboro Disease: Estimation of optimal time of vaccination by the Deventer formula. Proceedings of the $3 \mathrm{rd}$ meeting of working group 3 of COST action 839: passive protection and vaccination (current and future possibilities) in the presence of maternally derived antibody, Pulawy, pp: 21-28. 
De Wit, J.J., J.F. Heijmans, D.R. Mekkes and A. Van Loon, 2001. Validation of five commercially available ELISAs for the detection of antibodies against infectious bursal disease virus (serotype 1). Avian Pathol., 30: 543-549.

Efron, B., 1979. Rietz lecture-bootstrap methodsanother look at the jackknife. Ann. Stat., 7: 1-26.

FAO, 2001. FAOCLIM 2 (CD-ROM database): world-wide agroclimatic data, Rome.

FAO, 2008. FAOSTAT, Cited on 15 September 2008. Available from: http:/faostat.fao.org/site/291/default. aspx.

Intervet, 2003. Gumboro diseases - economical impact vaccination techniques. Poult. Focus Newsl., 3: 1-4.

Kouwenhoven, B. and J. Van den Bos, 1994. Control of very virulent infectious bursal disease (Gumboro Disease) in the Netherlands with more virulent vaccines. In: Kaleta, E.F. and U. Heffels-Redmann (Eds.), Proceedings of the international symposium on infectious bursal disease and chicken infectious anemia, Rauischholzhauzen, pp: 262-271.
Law, A.M. and W.D. Kelton, 2000. Simulation modeling and analysis, 3rd Ed, McGraw-Hill, New York, p. 800.

Lukert, P.D. and Y.M. Saif, 2003. Infectious bursal disease. In: Saif, Y.M., H.J. Barnes, J.R. Glisson, A.M. Fadly, L.R. McDougald and D.E. Swayne (Eds.), Diseases of poultry, 11th Ed, lowa State University Press, Ames., p: 1352.

Vaziry, A., D. Venne, D. Frenette, S. Gingras and A. Silin, 2007. Prediction of optimal vaccination timing for infectious bursal disease based on chick weight. Avian Dis., 51: 918-23. 